\title{
Phylogenetic and Preliminary Phenotypic Analysis of Yeast PAQR Receptors: Potential Antifungal Targets
}

\author{
Nancy Y. Villa $\cdot$ Patricia Moussatche • \\ Stephen G. Chamberlin • Anuj Kumar • \\ Thomas J. Lyons
}

Received: 25 October 2010/ Accepted: 7 September 2011/Published online: 19 October 2011

(c) The Author(s) 2011. This article is published with open access at Springerlink.com

\begin{abstract}
Proteins belonging to the Progestin and AdipoQ Receptor (PAQR) superfamily of membrane bound receptors are ubiquitously found in fungi. Nearly, all fungi possess two evolutionarily distinct paralogs of PAQR protein, which we have called the PQRA and PQRB subtypes. In the model fungus Saccharomyces cerevisiae, these subtypes are represented by the Izh2p and Izh3p proteins, respectively. S. cerevisiae also possesses two additional PQRA-type receptors called Izh1p and Izh4p that are restricted to other species within the "Saccharomyces complex". Izh2p has been the subject of several recent investigations and is of particular interest because it regulates fungal growth in response to proteins produced by plants and, as such, represents a new paradigm for interspecies communication. We demonstrate that $I Z H 2$ and IZH3 gene dosage affects resistance to polyene antifungal drugs. Moreover, we provide additional evidence that Izh2p and Izh3p negatively regulate fungal
\end{abstract}

Electronic supplementary material The online version of this article (doi:10.1007/s00239-011-9462-3) contains supplementary material, which is available to authorized users.

Present Address:

N. Y. Villa

Department of Medicinal Chemistry, College of Pharmacy, University of Florida, P.O. Box 100485, Gainesville,

FL 32610-0485, USA

P. Moussatche · S. G. Chamberlin · T. J. Lyons $(\bowtie)$

Foundation for Applied Molecular Evolution,

P.O. Box 13174, Gainesville, FL 32604, USA

e-mail: tlyons@ffame.org

\section{A. Kumar}

Departments of Molecular, Cellular, and Developmental Biology, Life Sciences Institute, University of Michigan, 210 Washtenaw Avenue, Ann Arbor, MI 48109-2216, USA filamentation. These data suggest that agonists of these receptors might make antifungal therapeutics, either by inhibiting fungal development or by sensitizing fungi to the toxic effects of current antifungal therapies. This is particularly relevant for pathogenic fungi such as Candida glabrata that are closely related to $S$. cerevisiae and contain the same complement of PAQR receptors.

Keywords PAQR · Osmotin · Fungi .

Saccharomyces cerevisiae - Antifungal · Polyene ·

Filamentation · Phylogeny · Progesterone .

Adiponectin

\section{Introduction}

The Progestin and AdipoQ Receptor (PAQR) family of proteins consists of a diverse group of evolutionarily related integral membrane receptors (Tang et al. 2005). Despite the fact that they have only recently been characterized, PAQRs have become the subject of intense scientific scrutiny due to their role in hormone signaling and in the etiology of metabolic disorders in humans (Yamauchi et al. 2003; Zhu et al. 2003). Fungal PAQRs have also garnered significant attention since it was discovered that S. cerevisiae PAQR (Izh2p) functions as a receptor for plant PR-5 proteins (Narasimhan et al. 2005), which are produced by plants as part of their innate immune response and potently inhibit fungal growth (Anzlovar and Dermastia 2003; Ferreira et al. 2007). It has been proposed that Izh2p (also called the osmotin receptor) mediates the PR-5 dependent induction of fungal apoptosis (Narasimhan et al. 2005), although an alternative-but not mutually exclusive-model has been proposed in which Izh2p negatively regulates filamentous growth (Kupchak et al. 2008). Either 
way, it is clear that Izh2p regulates fungal developmental programs in response to proteins produced by plants, suggesting that this agonist/receptor module represents an exciting new mode of interspecies communication.

PAQR proteins are unified by the presence of seven transmembrane domains (TM) and three short motifs $(\mathrm{A}-\mathrm{C})$ that are conserved in both sequence and location in the 7 TM PAQR core (see Fig. 10 for a description of the motifs). The presence of 7 TMs in the PAQR family has led to the proposal that these proteins are members of the G-Protein Coupled Receptor superfamily (GPCR) (Kulkarni et al. 2005; Lafon et al. 2006), although this assignment has been questioned based on detailed sequence and functional analysis (Smith et al. 2008). The PAQR family can be subdivided into three main classes (classes I, II, and III) based on distinctive physical characteristics that have been extensively described elsewhere (Smith et al. 2008). Class I PAQRs-which are archetypal PAQRs-are restricted to eukaryotes and include the human adiponectin receptors (AdipoR) (Yamauchi et al. 2003) as well as the yeast Izh2p and Izh3p. Class II receptors include the vertebrate membrane progesterone receptors (mPR) (Zhu et al. 2003) and are distinguished from Class I receptors solely by the presence of an additional transmembrane domain that is C-terminal to the PAQR core (Smith et al. 2008). Class III receptors are primarily prokaryotic but also include the human MMD protein (Rehli et al. 1995). Class III receptors are highly divergent from those in Classes I and II and can be easily distinguished by a more compact sequence and distinctive truncations of the conserved motifs (Villa et al. 2009). Some studies indicate that prokaryotic Class III proteins confer hemolytic activity onto their host strains (Baida and Kuzmin 1996; Chen et al. 2004; Robertson et al. 2006), leading to them being named hemolysin III (Hly3, HLY III). However, it is unclear if hemolytic activity is due directly to the PAQR proteinsespecially considering that human Class III receptors seem to play a role in macrophage maturation (Brauer et al. 2004; Rehli et al. 1995).

In this study, we present a detailed analysis of the PAQR family in fungi that reveals several interesting facts. First, fungi in the phylum Microsporidia are unique in that they posses Class III PAQRs but not Class I PAQRs. Moreover, microsporidian Class III receptors are more closely related to those of apicomplexans and actinobacteria than those of their sister group, the holozoans. Second, all other non-microsporidian fungi lack Class III receptors and instead seem to possess at least two Class I receptors-one in each of two distinct subgroups that we have called the PQRA and PQRB clades.

The flagship protein from the PQRA clade is the aforementioned Izh2p. The genomes of $S$. cerevisiae and closely related species-such as the human pathogen Candida glabrata - encode two other receptors belonging to this clade called Izh1p and Izh4p. Moreover, there seems to have been a variety of duplication events leading to numerous distinct PQRA homologs in Pezizomycotina, which is a fungal subphylum containing many medically and agriculturally important pathogens. Accordingly, there is great interest in studying the biochemistry and physiology of Izh2p and its homologs in the hopes that what is learned will lead to novel strategies for the treatment of recalcitrant fungal infections. The $\mathrm{PQRB}$ clade is represented in S. cerevisiae by Izh3p, a protein about which very little is known except that it seems to regulate the same intracellular signaling pathway as Izh2p, but does not seem to respond to any of the plant PR-5 proteins that have been tested (Kupchak et al. 2007; Lyons et al. 2004). Herein, we demonstrate that gene dosage of IZH2 and IZH3 affects both resistance to polyene antifungal drugs as well as fungal filamentation.

\section{Materials and Methods}

\section{Strains and Plasmids}

Except for the filamentous growth experiments, all strains are in the wild type BY4742 background. Construction of the $i z h 1 \Delta:: k a n M X 4$ [kanamycin resistance marker (kanMX4) (Wach et al. 1994)] and $i z h 3 \Delta:$ :natMX4 [nourseothricin resistance marker (natMX4) (Goldstein and McCusker 1999)] strains was previously reported (Lyons et al. 2004). Wild type, $n r g 1 \Delta:: \operatorname{kanMX} 4, n r g 2 \Delta:: \operatorname{kanMX} 4$, izh2 $\Delta:: k a n-$ MX4, izh $3 \Delta:: k a n M X 4$, and $i z h 4 \Delta:: k a n M X 4$ strains mutants were obtained from Euroscarf (http://web.uni-frankfurt.de/ $\mathrm{fb} 15 / \mathrm{mikro} /$ euroscarf/). Strains used for filamentation studies are described elsewhere and are in strain Y825 from the $\Sigma 1278$ b background (Jin et al. 2008).

All plasmids have been reported previously (Jin et al. 2008; Lyons et al. 2004). pIZH3 is a centromeric (single copy) plasmid containing the IZH3 gene with its native promoter and terminator $(\sim 1000$ bp upstream to $\sim 500 \mathrm{bp}$ downstream) and is used for complementation studies. $\mathrm{p} I Z H 1-G A L 1, \mathrm{p} I Z H 2-G A L 1, \mathrm{p} I Z H 3-G A L 1$, and $\mathrm{p} I Z H 4-$ $G A L 1$ are centromeric plasmids that express these genes using the $G A L 1$ promoter. GAL1-driven plasmids used for filamentation studies are in the pYES2/GS plasmid and are described elsewhere (Jin et al. 2008). GAL1-driven genes are induced by changing the carbon source from $2 \%$ glucose to $2 \%$ galactose.

Sequence Analysis

Sequences of PAQR receptors were obtained using BLAST to identify homologs in whole or partial genomes available on the National Center for Biotechnology Information 
(http://www.ncbi.nlm.nih.gov), the Joint Genome Institute (http://genome.jgi-psf.org), the Broad Institute (http:// www.broadinstitute.org), and the Sanger Institute (http:// www.sanger.ac.uk) websites. Most fungal PAQR genes do not contain introns, however, for those that do, it is well known that introns are sometimes incorrectly called in transcript databases. This leads to peptide sequences that are either incomplete or that contain spurious coding sequence. For this reason, we obtained the peptide sequences by BLASTing against genome sequences (TBLASTN) rather than peptide databases. The sequences can be found in the Supplementary Data. Sequence alignments and phylogenetic trees were prepared with the Seaview platform (Gouy et al. 2010) using Muscle 3.6 (Edgar 2004) to generate alignments and PhyML 3.0 (Guindon et al. 2009) to produce trees using the maximum likelihood (ML) method. Since, our sequence data consisted only of translated nucleotide data, we used the well-established WAG amino acid mutation matrix to model sequence evolution (Whelan and Goldman 2001). Standard model testing was performed, however, optimal trees did not show significant differences in crude topologies depending on model. The log files describing the actual parameters used for each tree, as well as the alignment file are included in the Supplementary material. Multiple sequence alignments reveal that Class I receptors contain multiple regions that align poorly. This includes large loop regions and the N-termini leading up to the first conserved PAQR motif. For tree generation, the presence or the absence of such non-aligning sequences in alignments had no effect on the overall topology of the trees or the conclusions drawn from them.

Hydropathy plots were generated with TopPredictII 1.2 using the Kyte-Doolittle algorithm and default parameters (Claros and von Heijne 1994). Hydropathy data was downloaded into a spreadsheet, sequences were aligned based on the multiple sequence alignments and the average hydropathy value at each position in the alignment was calculated. Sequence motifs were generated using WebLogo (Crooks et al. 2004).

\section{Phenotypic Studies}

All polyenes were acquired from Sigma-Aldrich. For all experiments, standard defined synthetic medium (SM) containing either $2 \%$ glucose or $2 \%$ galactose as a carbon source was used. For plating experiments, yeast strains were grown aerobically at $30^{\circ} \mathrm{C}$ in liquid medium overnight, serially diluted to $\mathrm{OD}_{600}$ of $1.0,0.1$, and 0.01 , and aliquots of $5 \mu \mathrm{l}$ were plated onto agar plates containing either vehicle (DMSO for nystatin, methanol for filipin, and pimaricin and water for amphotericin B) or the appropriate polyene stock solution. Over the course of experimentation, we discovered that the effective dose of each polyene was highly sensitive to the temperature of the agar medium when the drug was added-i.e., if the drug was added when the medium was $70^{\circ} \mathrm{C}$, much higher doses were required for toxicity than if the drug was added just before agar solidification at $50^{\circ} \mathrm{C}$. Thus, the effective doses of each polyene toward individual strains can vary from experiment to experiment depending on how the plates are made. Moreover, polyene stocks were used immediately after preparation to avoid drug decomposition.

For complementation studies, the wild type and $i z h 3 \Delta$ mutant strains were transformed with pIZH3 or the empty expression vector control. For overexpression studies, the wild type strain was transformed with pGAL1 plasmids containing $I Z H 1, I Z H 2, I Z H 3$, or $I Z H 4$ or the empty expression vector control. Cells were spotted onto the appropriate solid medium with or without nystatin as described above.

Filamentation studies were performed as previously described (Jin et al. 2008). In brief, cells were grown on plates containing low nitrogen synthetic defined medium with $1 \%$ butanol (v/v) to induce filamentous growth. For overexpression studies $2 \%$ galactose was included instead of glucose to induce gene expression. Strains were streaked for single colonies and filamentous morphology was assessed by microscopy.

\section{Results and Discussion}

\section{Phenotypic Analysis}

Because of the simplicity and tractability of $S$. cerevisiae as a eukaryotic model system, there is increasing interest in understanding the biology of yeast PAQR receptors as models for the entire PAQR family (Kupchak et al. 2007; Narasimhan et al. 2005; Villa et al. 2009). Despite a growing body of literature regarding this protein family in yeast, there is still little understanding of the precise function of these receptors. Consequently, we have undertaken the phenotypic analysis of $I Z H 2$ and $I Z H 3$.

Izh2p, Izh3p, and Polyene Resistance

It has been reported that yeast strains lacking Izh2p are resistant to the polyene antifungal drug nystatin when added to media at $100 \mathrm{U} / \mathrm{ml}$ (Karpichev et al. 2002). In our hands, $100 \mathrm{U} / \mathrm{ml}(25 \mu \mathrm{g} / \mathrm{ml})$ nystatin was lethal to all strains tested and we were still unable to detect increased resistance to nystatin in the $i z h 2 \Delta$ strain at $10 \mathrm{U} / \mathrm{ml}$ (2.5 $\mu \mathrm{g} / \mathrm{ml})$ (Fig. 1a). This discrepancy in nystatin toxicity between our results and reported values may be due to (a) variability in the potency of nystatin batches, (b) the 


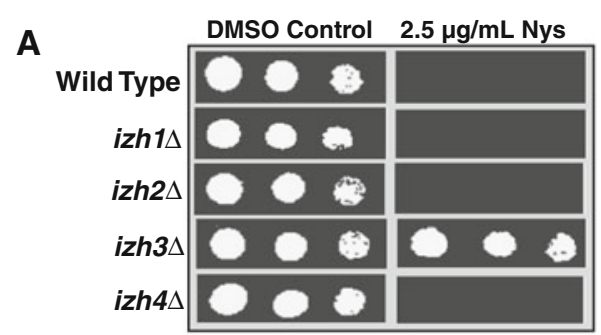

B

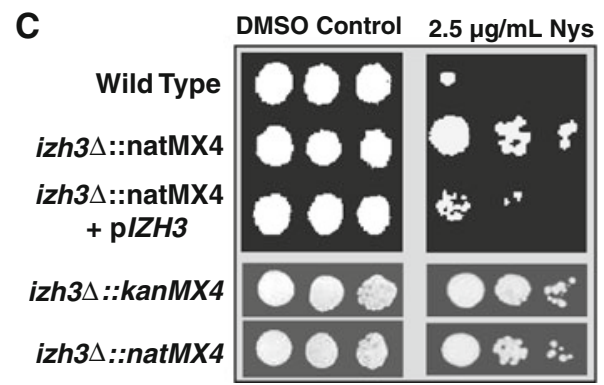

Fig. 1 Polyene resistance. Plates contain either vehicle or vehicle + polyene treatment. a Sensitivity of various strains to $2.5 \mu \mathrm{g} / \mathrm{ml}$ nystatin, b sensitivity of wild type, $i z h 2 \Delta:: k a n M X 4$ and $i z h 3 \Delta::$ kanMX4 strains to $0.5 \mu \mathrm{g} / \mathrm{ml}$ nystatin, $\mathbf{c}$ complementation of the nystatinresistance phenotype for $i z h 3 \Delta$. The transformation of the $i z h 3 \Delta:$ :natMX4 strain with a single copy plasmid containing the IZH3 gene driven by its native promoter ( $\mathrm{pIZH3}$ ) results in wild type-like sensitivity to $2.5 \mu \mathrm{g} / \mathrm{ml}$ nystatin (top). Deletion of the IZH3 gene with either the kanMX4 or natMX4 markers results in similar resistance to

temperature at which nystatin was added before the plates were poured (see "Materials and Methods"), (c) differences in the wild type strain background, or (d) small differences in the $I Z H 2$ deletion construct. Nevertheless, we were able to detect slight but reproducible resistance at $0.5 \mu \mathrm{g} / \mathrm{ml}(2.6 \mathrm{U} / \mathrm{ml})$ (Fig. 1b). Neither the deletion of $\mathrm{IZHI}$ nor of $\mathrm{IZH} 4$ resulted in nystatin resistance (Fig. 1a).

Unlike the other mutants, the $i z h 3 \Delta$ strain displays significant resistance to $10 \mathrm{U} / \mathrm{ml}(2.5 \mu \mathrm{g} / \mathrm{ml})$ nystatin on solid medium (Fig. 1a). This phenotype has not been reported before. It is possible that the resistance of the $i z h 3 \Delta$ mutant was due to the nourseothricin resistance marker (natMX4) used to delete the IZH3 gene. However, the same resistance to $2.5 \mu \mathrm{g} / \mathrm{ml}$ nystatin was obtained when we used the $i z h 3 \Delta$ mutant from the Saccharomyces Deletion Collection in

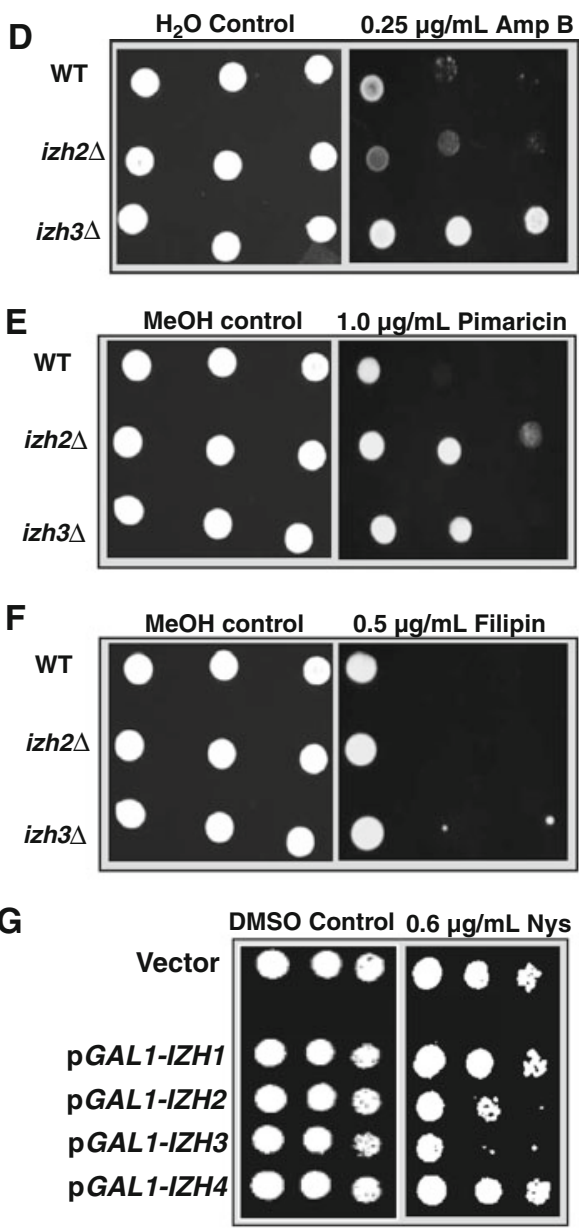

$2.5 \mu \mathrm{g} / \mathrm{ml}$ nystatin (bottom). d-f Sensitivity of wild type, $i z h 2 \Delta:$ :kanMX4 and $i z h 3 \Delta:: k a n M X 4$ strains to $0.25 \mu \mathrm{g} / \mathrm{ml}$ amphotericin B (Amp B) (d), $1.0 \mu \mathrm{g} / \mathrm{ml}$ pimaricin (e), or $0.5 \mu \mathrm{g} / \mathrm{ml}$ filipin (f). g Overexpression of $I Z H 1, I Z H 2, I Z H 3$, and $I Z H 4$ in a wild type strain using a galactose inducible promoter. PAQR overexpressors (pGAL1-IZH) are compared to a wild type strain carrying empty expression vector. $I Z H 2$ and $I Z H 3$ overexpressors are more sensitive to $0.6 \mu \mathrm{g} / \mathrm{ml}$ nystatin than the wild type strain

which IZH3 was deleted using the kanMX4 marker (Fig. 1c). Moreover, the resistance of the $i z h 3 \Delta$ strain to $2.5 \mu \mathrm{g} / \mathrm{ml}$ nystatin could be complemented by transforming the strain with the $I Z H 3$ gene driven by its native promoter and terminator on a single copy centromeric plasmid (pIZH3).

Deletion of $I Z H 2$ or $I Z H 3$ also results in resistance to other polyene antifungals, including amphotericin B (Fig. 1d) and pimaricin (also called natamycin) (Fig. 1e). Their resistance to amphotericin B mirrors that of nystatin, with $i z h 3 \Delta$ being more resistant than $i z h 2 \Delta$. This might be expected considering that nystatin and amphotericin $\mathrm{B}$ work via similar mechanisms (Lopes et al. 2004). On the other hand, $i z h 2 \Delta$ is more resistant to pimaricin than $i z h 3 \Delta$. This is perhaps not surprising since pimaricin is postulated 
to have a slightly different mechanism of action than nystatin and amphotericin B (te Welscher et al. 2008). Finally, neither strain is resistant to a fourth polyene, filipin (Fig. 1f). Again, this is not surprising since filipin is the only non-amphoteric compound of the group and is believed to function very differently than the others (Lopes et al. 2004).

We also observed that overexpression of both $I Z H 2$ and IZH3 - but not IZHI and IZH4-conferred sensitivity to levels of nystatin $(0.6 \mu \mathrm{g} / \mathrm{ml})$ that had no effect on the growth of the wild type strain (Fig. 1g) (discrepancies between nystatin potency in Fig. $1 \mathrm{~g}, \mathrm{~b}$ are likely due to the temperature of the media when the antifungal agent was added, however, it is also possible that the discrepancy is due to the fact galactose was used as the carbon source in Fig. 1g and glucose in Fig. 1b.)

Izh2p, Izh3p, and the Regulation of Filamentous Growth

We previously reported that overexpression of or ligandmediated activation of heterologously expressed PAQRs in yeast (including Izh2p) negatively controlled the activity of a pair of transcriptional repressors known as $\mathrm{Nrg} 1 \mathrm{p}$ and Nrg2p (Garitaonandia et al. 2009; Kupchak et al. 2007). $\mathrm{Nrg} 1 \mathrm{p}$ and $\mathrm{Nrg} 2 \mathrm{p}$ are known to play an important role in the inhibition of the developmental program known as filamentous growth. This is true in $S$. cerevisiae as well as the pathogen C. albicans (Murad et al. 2001). This leads to the following question: if overexpression of PAQRs activate the pathway leading to $\mathrm{Nrg} 1 \mathrm{p} / \mathrm{Nrg} 2 \mathrm{p}$, then their overexpression should repress filamentous growth. Accordingly, as shown in Fig. 2, the overexpression of either Izh2p or Izh3p represses filamentous growth in an assay developed by Jin et al. (2008). Conversely, it follows that, if Izh2p and Izh3p overexpression represses filamentation, then reduced gene dosage should hyper-activate filamentation. Indeed, the $i z h 3 \Delta$ mutant strain demonstrates a hyperfilamentous phenotype (Fig. 2). However, the $i z h 2 \Delta$ mutant displays a wild type-like phenotype with respect to filamentous growth - a finding that has been confirmed by others (Narasimhan et al. 2005). The lack of an effect in the $i z h 2 \Delta$ strain can be explained by the fact that it is a receptor for proteins produced by plants. Therefore, its effect on filamentation only manifests when its agonist is present or the receptor is overexpressed-thereby activating the signaling machinery. Its deletion would not be expected to have any effect in the absence of agonist. The situation is different for Izh3p since its agonist is unknown and may be an endogenous molecule.

In summary, Izh2p and Izh3p seem to play an important, but still poorly understood, role in fungal development and resistance to polyene antifungal drugs. Consequently, these

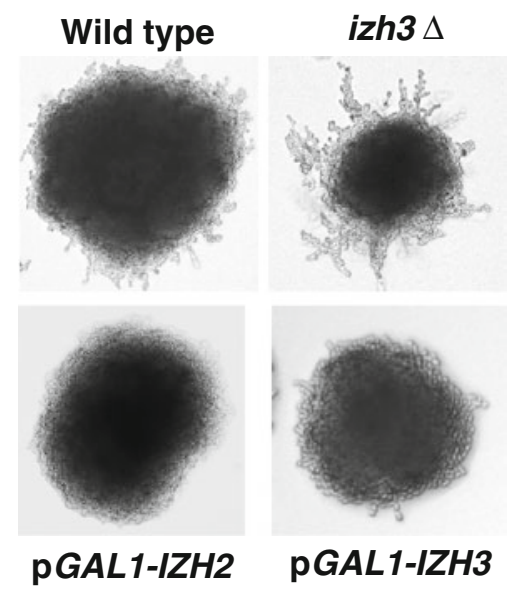

Fig. 2 Role in filamentation. When grown on synthetic defined plates containing $1 \%$ butanol the wild type strain undergoes filamentous growth. The izh $3 \Delta$ mutant displays a hyperfilamentous phenotype while strains overexpressing either $I Z H 2$ or $I Z H 3$ display a hypofilamentous phenotype

data, along with data from other groups (Narasimhan et al. 2005), suggest that PAQRs represent important new antifungal targets.

\section{Phylogenetic and Evolutionary Analyses}

In order to be regarded as legitimate antifungal targets, it is critical that target genes be conserved in a diverse array of medically or agriculturally important fungi. It is for this reason that a detailed phylogenetic and evolutionary analysis of the PAQR family in fungi and related eukaryotes was performed.

\section{Classes I and III Receptors in Eukaryotes}

Figure 3 is a bootstrapped phylogenetic tree of all known non-metazoan and non-fungal eukaryotic PAQRs and a representative subset of fungal and metazoan receptors. The bootstrap value $(500 / 500)$ at the base of the tree unequivocally demonstrates the conclusion that PAQR receptors can be subdivided into two fundamental classes (Receptors in Classes I and II are similar, and may represent a single class of receptor. However, the true evolutionary history of Class II receptors is still largely unexplored and these receptors are not included in Fig. 3 because there are no members of this class present in any fungal genome. As such, Class II receptors are excluded from this study). BLAST searches of eukaryotic genomes reveals that both Classes I and III receptors are broadly distributed among eukaryotic taxa, although there are a variety of organisms whose genome does not encode any PAQR receptor. Examples of complete genomes lacking PAQRs altogether include those of Bigelowiella natans 


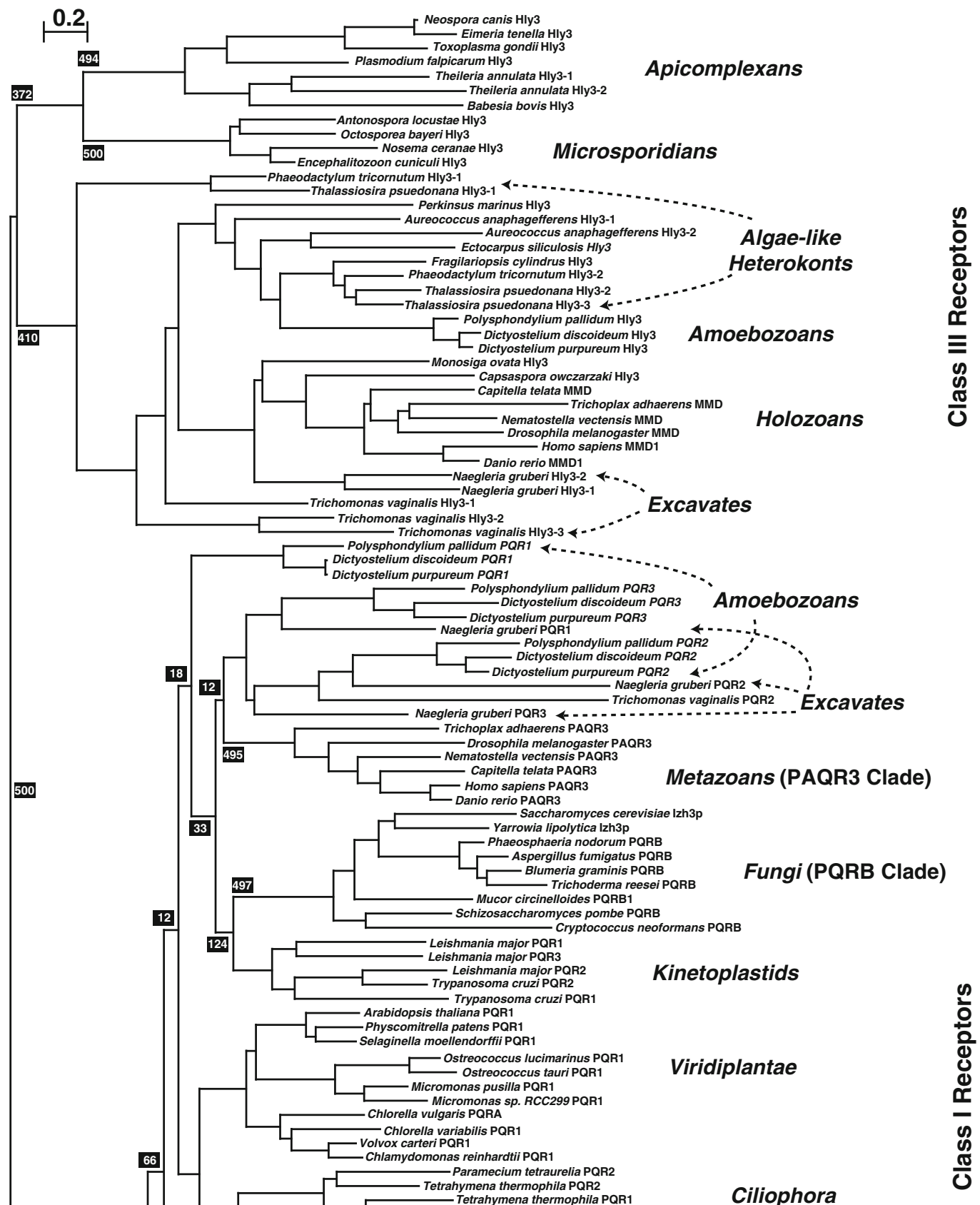

Fungi-like

Heterokonts

Fungi (PQRA Clade)

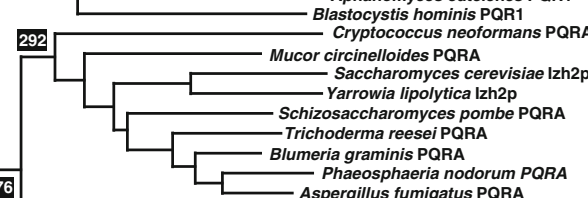

276

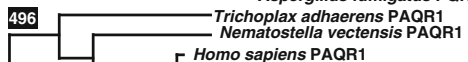

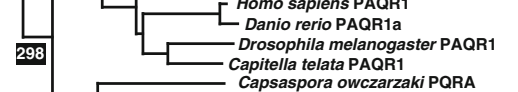

Holozoans (PAQR1 Clade)

Capsaspora PACarzaki PQRA 
4 Fig. 3 Phylogenetic analysis of eukaryotic PAQRs. A bootstrapped phylogenetic tree of eukaryotic receptors from Classes I and III. Bootstrap values (black shaded boxes) indicate the number of times per 500 trees generated a particular grouping is made and only those values of importance for the conclusions are shown. Branch lengths are proportional to evolutionary distance and the scale bar indicates the number of substitutions per site. Each clade is accompanied by a generalized taxonomic description of the organisms found within it. Taxonomic groupings are clarified with arrows if the distribution of sequences from a particular grouping does not correspond to a single clade or if individual sequences within a clade do not belong to the generalized grouping of that clade

(Rhizaria), Cryptosporidium parvum (Apicomplexa) as well as Guillardia theta and Hemiselmis andersenii (Cryptophyta), although it is possible that the absence of PAQRs in these genomes is due to undetected gaps in the genomic sequences. The sparse sampling of eukaryotic diversity also makes it impossible to draw any conclusions about what type of protist has PAQRs and why.

What can be said is that-with the exception of species in the phylum Microsporidia-Class I receptors have been found in all species in the opisthokont group sequenced to date, including all non-microsporidian fungi, the choanozoans Monosiga ovata and Monosiga brevicollis, the filasterean Capsaspora owczarzaki and all metazoans.

On the other hand, Class III receptors are present in microsporidians, but not in any other fungi. Class III receptors have, so far, been found in all filozoans (Filasterea and Metazoa) as well as in the choanozoan, M. ovata. Unexpectedly, no Class III receptor can be detected in the complete $M$. brevicollis genome, suggesting that either $M$. brevicollis is markedly different from $M$. ovata with respect to the complement of PAQRs or that there may be undetected gaps in the $M$. brevicollis genome sequence.

\section{Phylogenetic Analysis of the Fungal Class I PAQRs}

Figure 4 shows a bootstrapped phylogenetic tree of highly conserved Class I PAQR receptors from a representative set of fungi and metazoa. Figure 4 can be thought of as a magnification of Fig. 3 to focus on fungal and metazoan proteins in the Class I clade. The take home message of Fig. 4 is that fungal Class I receptors reliably cluster into two distinct subgroups. Genome searches also indicate that every fungal genome encodes at least one Class I PAQR from each subgroup, which we have named the PQRA and PQRB clades, although it appears as though most fungi possess only one paralog from each clade. Exceptions to this rule include (a) species in the subphylum Mucormycotina, which possess multiple PQRB paralogs, (b) species in either the subphylum Pezizomycotina, the "Saccharomyces complex" and some species in Agaricomycetes which may possess alternative PQRA subtypes that are distinct from the main PQRA paralog, and (c) the chytrid fungus Batrachochytrium dendrobatidis, which has two receptors that cannot be easily categorized based on either phylogenetic analysis or comparison of conserved motifs (see below).

\section{Multiple PQRA Paralogs in Pezizomycotina} and Basidiomycota

To get a clearer picture of the relationships between various PQRA homologs in fungi, we generated a separate bootstrapped phylogenetic tree with a larger selection of PQRA proteins from fungi (Fig. 5). This tree can be thought of as a magnification of the tree in Fig. 4 with a focus on the fungal PQRA clade and the addition of A1, A2, A3, A4, and A5 homologs from Pezizomycotina, and alternative PQRA proteins from the "Saccharomyces complex" and Agaricomycetes. The A1 homologs are universally found in species in Pezizomycotina. Moreover, A1 homologs are monophyletic in the tree in Fig. 5 (bootstrap value 83/100) and the clustering individual proteins in this clade conforms to known taxonomic groupings, suggesting that the A1 homolog is the "original" PQRA in the subphylum. The A1 clade forms a sister group with PQRA proteins from Taphrinomycotina (bootstrap value 37/100), while the A3 clade of proteins joins these two groups at the next branch point (bootstrap 46/100). PQRA proteins from Saccharomycotina form a sister group to the A1/TaphrinomycotinalA3 clade (bootstrap value 52/100), while the A2, A4, and A5 paralogs reside outside this grouping, but within the grouping that contains all species in the phylum Ascomycota (bootstrap value 49/100). All PQRA homologs from Ascomycota predictably form a sister group with PQRA homologs from Basidiomycota. Relatively low bootstrap values preclude us from drawing firm conclusions about how alternative PQRA homologs from Pezizomycotina relate to other fungal PQRA proteins. However, the monophyly of all PQRA proteins in the subkingdom Dikarya (bootstrap value 57/100) suggests that the A2, A3, A4, and A5 homologs did not enter the Pezizomycotina lineage via other kingdoms and may have originated in fungi. The precise number of PQRA subtypes in Pezizomycotina and how they are interrelated is not a critical aspect of this study. What is important is that the A2, A3, A4, and A5 homologs are not universally found in all species in the subphylum and even closely related species may have different complements of PQRA proteins. Based on the limited number of complete genome sequences available (and the possibility that some of these sequences may contain undetected gaps), it is not yet possible to definitively categorize these subtypes and make predictions about which subtypes may be found in each species within Pezizomycotina. 


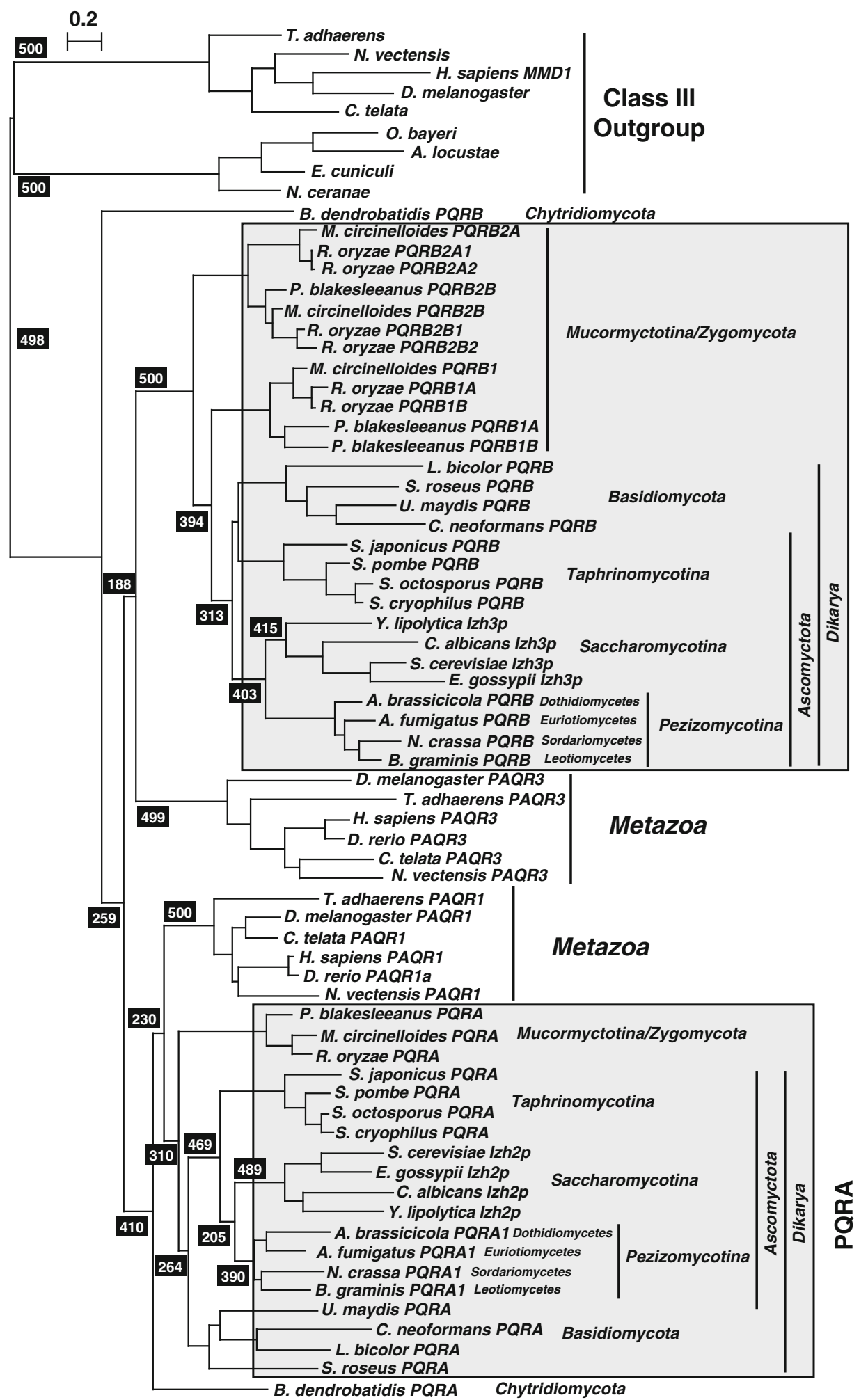

Fig. 4 Phylogenetic analysis of fungal and metazoan Class I PAQRs. A bootstrapped phylogenetic tree of selected fungal and metazoan Class I PAQRs. This tree shows the unequivocal presence of two distinct clades in both fungi and metazoans. Bootstrap values (black shaded boxes) indicate the number of times per 500 trees generated a particular grouping is made and only those values of importance for the conclusions are shown. Branch lengths are proportional to evolutionary distance and the scale bar indicates the number substitutions per site. Gray shading indicates the PQRA and PQRB clades of fungal receptors. Metazoan and fungal Class III receptors are included as an outgroup 
Some species in the class Agaricomycetes (subphylum Agaricomycotina, phylum Basidiomycota) seem to possess two PQRA homologs: one (A1A) that clusters with other PQRA proteins from Agaricomycetes (bootstrap value 94/100) and a second (A1B) that clusters with PQRA proteins from Microbotryomycetes (subphylum Pucciniomycotina, phylum Basidiomycota) (bootstrap value 87/100). This indicates that the A1B homolog in Agaricomycetes may have arisen through lateral gene transfer from Microbotryomycetes. More data is needed to confirm this hypothesis.

\section{PAQRs from the "Saccharomyces Complex"}

The $S$. cerevisiae genome encodes three members of the PQRA clade (Izh1p, Izh2p, and Izh4p) and one member of the PQRB clade (Izh3p). Not surprisingly, Saccharomyces pastorianus contains two homologs of each IZH protein: i.e., two Izh1p, two Izh2p, two Izh3p, and two Izh4p. One homolog in each pair clusters with its ortholog from $S$. cerevisiae and the other clusters with its ortholog in Saccharomyces uvarum CBS 7001 (a.k.a. Saccharomyces bayanus). This is consistent with the fact that S. pastorianus is an allopolyploid interspecies hybrid of $S$. cerevisiae and Saccharomyces eubayanus, which, among Saccharomyces species, is most closely related to $S$. uvarum (Libkind et al. 2011).

\section{Izhlp and Izh4p are Related by a Whole Genome Duplication Event}

Figure 6 shows a bootstrapped phylogenetic tree of all PQRA proteins from species in the subphylum Saccharomycotina with representative proteins from Pezizomycotina forming an outgroup. This tree can be thought of as a magnification of the tree in Fig. 5 with a focus on PQRA proteins from Saccharomycotina. Izh1p-like and Izh4p-like proteins are monophyletic in this tree, indicating a close relationship between these two proteins (bootstrap value 100/100). Comparative genomic analysis reveals that all species possessing Izh4p homologs belong to the genera Saccharomyces, Nakaseomyces, or Naumovozyma, which are closely related (Kurtzman 2003) and descend from the whole genome duplication (WGD) event known to have occurred in the Saccharomyces lineage approximately 100 million years ago (Gordon et al. 2009). Therefore, it is reasonable to postulate that Izh4p arose during the WGD event from another PQRA homolog. Genomic analysis reveals a large degree of synteny between the chromosomal regions containing $I Z H 4$ and $I Z H I$ in yeasts that descend from the WGD event (Fig. 7), confirming identity of IZHI as the ohnolog of $\mathrm{IZH} 4$.
The multiple sequence alignment in Fig. 8 shows that Izh4p-like proteins are highly divergent from other members of the PQRA clade (in this case Izh1p proteins), with the motifs that are considered "diagnostic" of PAQRs (motifs A-C) being barely recognizable. Using the tree in Fig. 6, we calculated the branch lengths from the branch point that includes all Izh1p and Izh4p proteins (branch point A, Fig. 6) to each protein in this clade and then averaged these lengths for all Izh4p proteins and all Izh1p proteins. The average distance from the branch point for all Izh1p proteins is $0.89 \pm 0.19$, while the average distance for all Izh4p proteins is $2.16 \pm 0.32$ (see Fig. 6, box 1). This $\sim 2.4$ difference is significant and indicates a high substitution rate for Izh4p relative to Izh1p soon after the duplication event that gave rise to their respective genes. Subsequent to gene duplication events, it is common for one gene to retain the function of the parent (Izh1p) and the other to experience relaxed selection (Izh4p) (Lynch and Conery 2000). Indeed, Lynch and Conery predicted that the average duplicated gene would last only $\sim 4$ million years before it is silenced or removed. It is not surprising, then, that no IZH4 gene is present in the genome of Vanderwaltozyma polyspora, a member of the earliest diverging clade of the post-WGD yeasts (Scannell et al. 2007). However, this does not explain why other post-WGD species retain IZH4 after 100 million years. More importantly, ESTs from S. cerevisiae and S. pastorianus confirm that IZH4 is expressed (Miura et al. 2006; Yoshida et al. 2007), suggesting that they have not been silenced. In addition, a comparison of the average distances for each receptor group from the earliest branch point that includes all Saccharomyces species (branch point B, Fig. 6) suggests that Izh4p is evolving only $25-30 \%$ faster than either Izh1p or Izh2p in this genus (see Fig. 6, box 2). Furthermore, $t$ tests revealed that the increase in substitution rate for Izh4p relative to Izh1p and Izh2p is not statistically significant. Thus, while the Izh4p group as a whole is changing faster than the Izh1p group, the rate of change in Izh4p relative to Izh1p seems to have slowed down considerably in Saccharomyces spp. The fact that Izh4p still exists, is expressed and has not changed significantly faster than Izh1p-like proteins in the 20 million or so years since $S$. cerevisiae diverged from $S$. uvarum [date of divergence estimated in (Kellis et al. 2003)] suggests that Izh4p may have acquired a new function along the way. In support of this hypothesis, we previously demonstrated that Izh4p overexpression can activate the same downstream signaling pathway as Izh1p, Izh2p, and Izh3p, indicating that Izh4 $p$ is functional and retains some signaling capability (Kupchak et al. 2007; Lyons et al. 2004). Because it appears as though Izh1p retained the function of the ancestor to Izh1p and Izh4p, we have proposed that all 


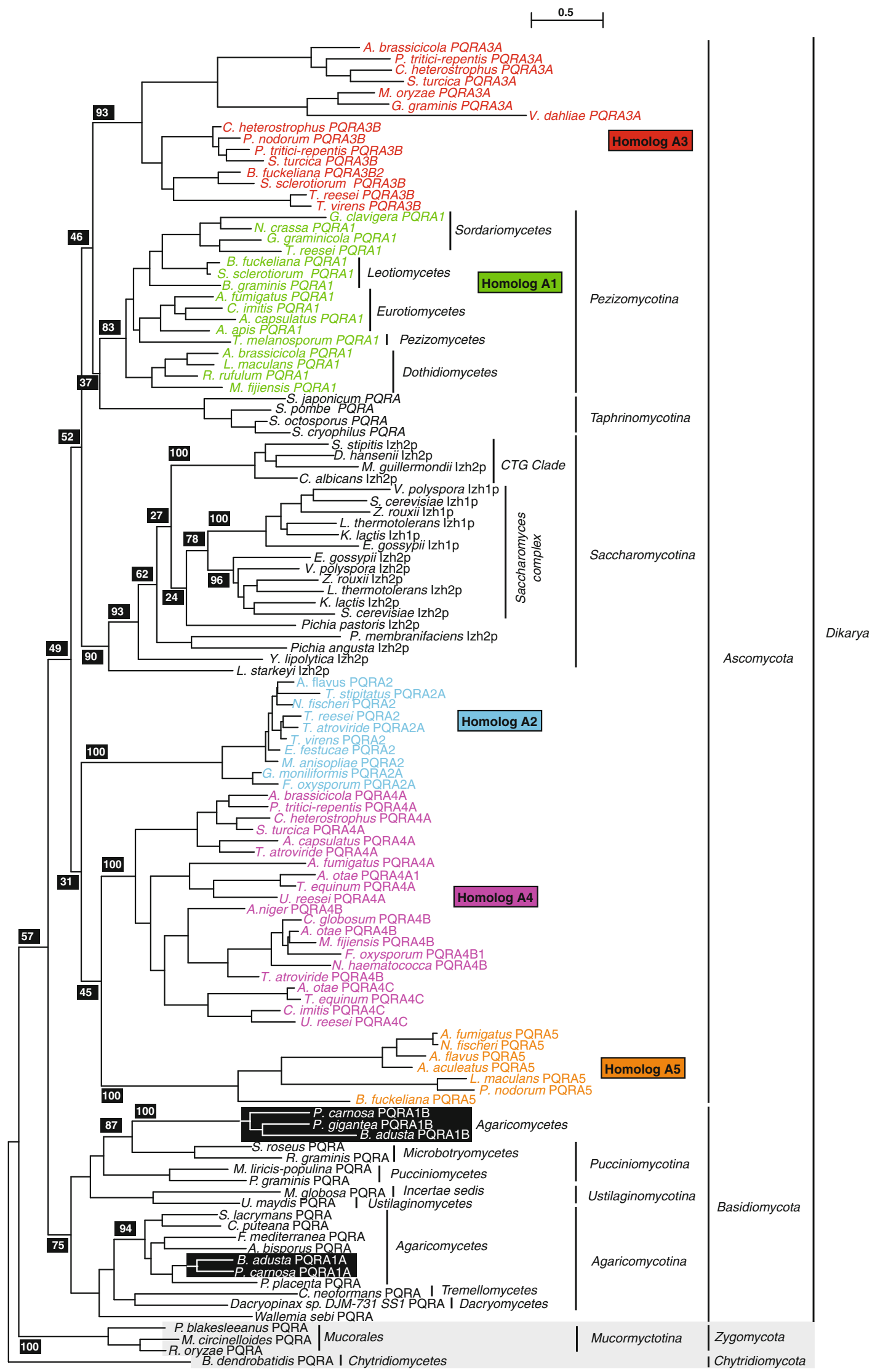


Fig. 5 Phylogenetic analysis of PQRA proteins from fungi. A bootstrapped phylogenetic tree of PQRA homologs from fungi. The tree shows the relationship between alternative PQRA homologs in Pezizomycotina, the "Saccharomyces complex" and Agaricomycetes and the main PQRA subtype. Bootstrap values (black shaded boxes) indicate the number of times per 100 trees generated a particular grouping is made and only those values of importance for the conclusions are shown. Branch lengths are proportional to evolutionary distance and the scale bar indicates the number substitutions per site. Each sequence is accompanied by a taxonomic description of the organism in which it is found

Izh1p-like proteins within the "Saccharomyces complex", be referred to as Izh1p.

\section{Izhlp-like and Izh2p-like Proteins are Likely Related by Another Duplication Event}

The phylogenetic trees in Figs. 5 and 6 clearly demonstrate that Izh1p- and Izh2p-like proteins are monophyletic in trees including all receptors from hemiascomycete fungi (bootstrap values of 78/100 and $81 / 100$, respectively). This suggests a close relationship between Izh1p- and Izh2p-like proteins. Izh1p-like proteins are present in all members of the "Saccharomyces complex" but absent from all hemiascomycete genera in what is known as the "CTG clade" [as defined in (Fitzpatrick et al. 2006)]. Izh1p-like proteins are also absent from the more distantly related Yarrowia lipolytica, Pichia membranifaciens, Pichia pastoris, Pichia angusta, and Lipomyces starkeyi. The simplest explanation is that Izh2p and Izh1p arose from a duplication event that occurred after the divergence of the "Saccharomyces complex" from the CTG clade, which has been estimated to have occurred 150-180 million years ago (Massey et al. 2003). A comparison of the distance from the branch point that includes both Izh1p- and Izh2plike proteins from the "Saccharomyces complex" (branch point C, Fig. 6) to the branch points containing each group individually (branch point D, Fig. 6) suggests a higher substitution rate for the Izh1p group immediately following the duplication event. Indeed, the average distance from branch point $C$ for all Izh1p-like proteins $(1.33 \pm 0.19)$ is significantly higher than for all Izh2p-like proteins $(1.01 \pm 0.14)$ (see Fig. 6 , box 3). However, there is no significant difference between the average distances of Izh1p- and Izh2p-like proteins from branch point D (see Fig. 6, box 4). Thus, like Izh4p, Izh1p seems to have experienced an initial period of relaxed selection followed by increased selective pressure after the emergence of Saccharomyces spp., most likely due to the acquisition of some new function. Since, Izh2p appears to have retained the function of the ancestor of Izh1p and Izh2p, we propose that all PQRA-like proteins from species outside the "Saccharomyces complex" but within the subphylum Saccharomycotina be referred to as Izh2p.

\section{The Split Between Izhlp/Izh2p/Izh4p and Izh3p is Ancient}

Like most fungi, $S$. cerevisiae only possesses one receptor in the PQRB clade, which is named Izh3p. Figure 9 shows aligned hydropathy plots for proteins from the PQRA clade (Fig. 9a) and PQRB clade (Fig. 9b), demonstrating the presence of $7 \mathrm{TMs}$ and the location of the three conserved motifs (A-C). The most distinctive characteristic of Izh3plike proteins is the presence of long $\mathrm{N}$-terminal extensions ( $\sim 300$ a.a. compared with $\sim 100$ a.a. for PQRA proteins). It is intriguing that, while nearly all members of the PQRB clade possess this extension, there does not seem to be any conservation of amino acid sequence even among closely related species. The function of this domain is unknown and BLASTing the sequences of these extensions against peptide databases has yet to reveal similarity to any other protein family.

Using alignments containing all fungal PQRA (115 sequences, A1 paralog only) or PQRB proteins (124 sequences) from the Supplementary data, we generated probability-based consensus motifs for the two clades. The three characteristic PAQR motifs and the subtle differences between fungal PQRA-like and PQRB-like receptors are shown in detail in Fig. 10. It should be noted that Izh3p and several other PQRB proteins from Saccharomycetaceae species include a long non-conserved loop region in the middle of motif $\mathrm{C}$. This loop region has been removed from the sequence of Izh3p-like proteins to produce the motifs shown in Fig. 10. The tryptophan or phenylalanine at position 4 in motif $\mathrm{C}$ is generally the most reliable way to distinguish PQRA proteins from PQRB proteins.

The presence of PQRA-like and PQRB-like proteins in all non-microsporidian fungi suggests that the last common ancestor of both clades may have preceded the emergence of fungi, which has been proposed to have occurred over 600 million years ago, at the very least (Taylor and Berbee 2006). However, the two PAQRs encoded by the genome of the basal chytrid fungus $B$. dendrobatidis complicate the issue. One (PQRA) strongly clusters with the PQRA clade in Fig. 4 (bootstrap value of 410/500 replicates), however, it lies outside the clade that contains both non-chytrid fungal and holozoan PQRA proteins. The other chytrid receptor (PQRB) does not show a strong affiliation with either the PQRA clade or the PQRB clade. The ambiguous clustering of the chytrid receptors is elucidated if one looks at the diagnostic motifs. For example, PQRA from $B$. dendrobatidis has PQRA-like motifs A and B, but a PQRB-like motif C. Along the same lines, $\mathrm{PQRB}$ from $B$. dendrobatidis has motifs A and B with both PQRA- and PQRB-like characteristics and a motif $\mathrm{C}$ with $\mathrm{PQRB}$-like characteristics (see Fig. 10). Because B. dendrobatidis is the only chytrid for which the genome sequence is available, it is impossible to 


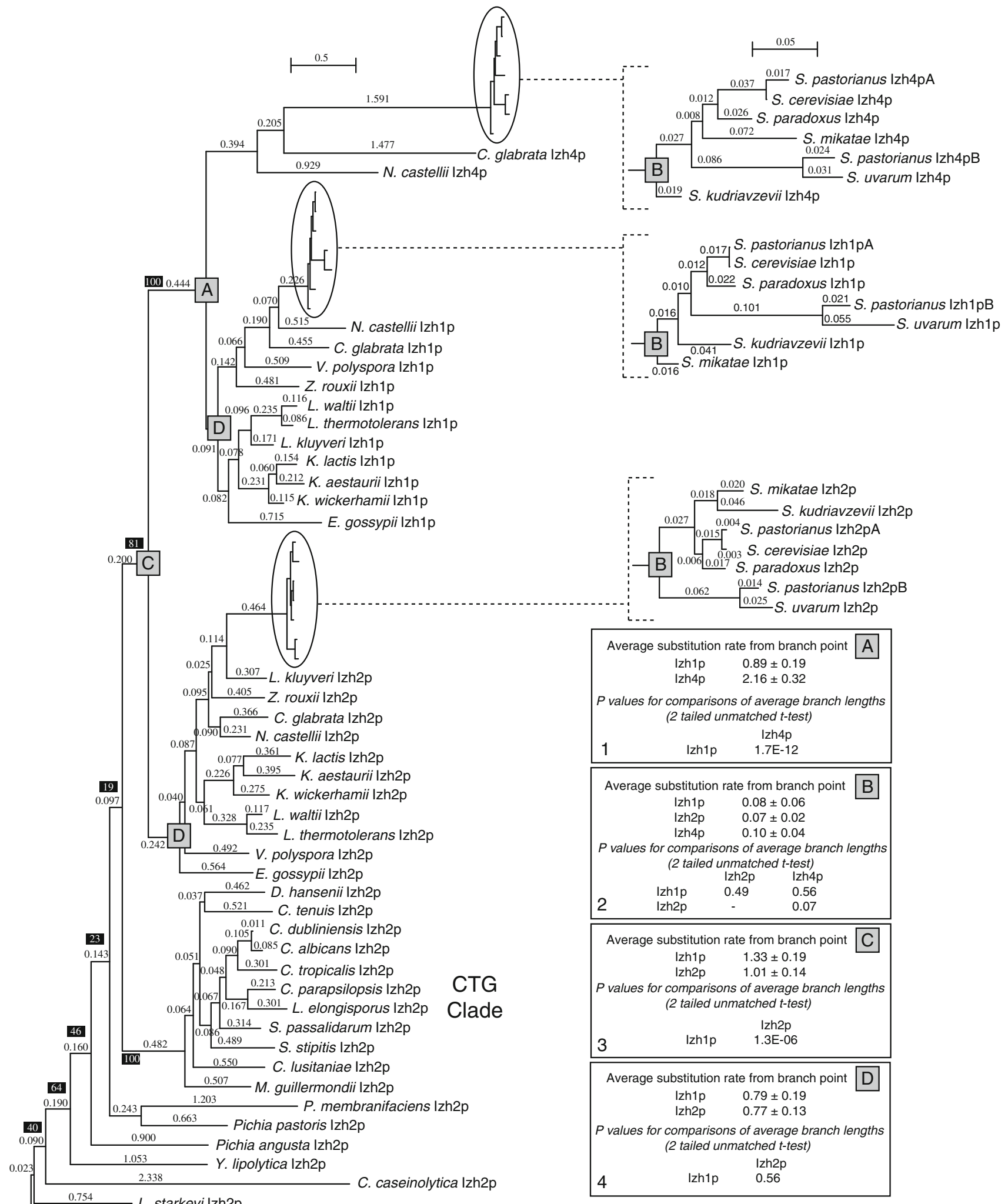

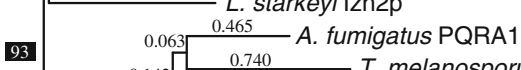

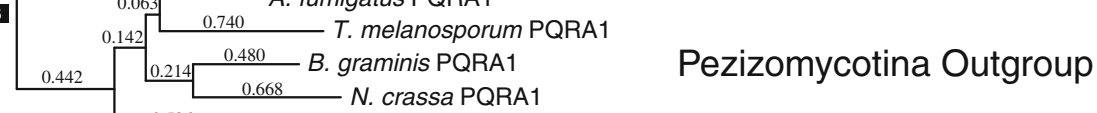

0.586 P. nodorum PQRA1 
4 Fig. 6 Phylogenetic analysis of Class I PAQRs from the subphylum Saccharomycotina. A bootstrapped phylogenetic tree of hemiascomycete Class I PAQRs. Representative sequences from Pezizomycotina are included as an outgroup. Bootstrap values (black shaded boxes) indicate the number of times per 100 trees generated a particular grouping is made and only those values of importance for the conclusions are shown. Subtrees for species in the genus Saccharomyces are magnified $\times 10$ on the right side of the figure. Branch lengths are proportional to evolutionary distance and the scale bar indicates the number substitutions per site. Boxes 1-4 show comparisons of average branch lengths for various protein groups from particular branch points (gray boxes) as well as the results of $t$ tests to determine the significance of differences in average branch lengths

state whether its receptors are characteristic of chytrid PAQRs or are an aberration. Consequently, one possibility is that PAQRs from this basal fungus resemble the ancestor(s) of both the PQRA and PQRB clades, suggesting that these clades arose after chytrids diverged from the rest of the fungal lineage, which has been estimated to have occurred approximately 500 million years ago (Chabasse 1998). Another possibility is that the chytrid receptors arose through chimerization of $\mathrm{PQRA}$ and $\mathrm{PQRB}$ receptors. Based on current genomic information, these two possibilities cannot yet be distinguished. Therefore, the simplest conclusion is that the chytrid receptors are outliers and the split between the PQRA and PQRB clades precedes the emergence of fungi.

The conclusion that the split between the PQRA and PQRB clades precedes the emergence of fungi is supported by a similar cladistic split that has previously been noted in metazoan Class I receptors (Garitaonandia et al. 2009). All metazoans have at least two types of Class I PAQR - one that resembles human PAQR1 and one that resembles human $P A Q R 3$. The distinguishing characteristic of metazoan PAQR1-like proteins is an $\mathrm{HxxFH}$ in motif $\mathrm{C}$, making it analogous to the PQRA clade in fungi. The distinguishing characteristic of metazoan PAQR3-like proteins is an $\mathrm{HxxWH}$ in motif $\mathrm{C}$, making it analogous to the $\mathrm{PQRB}$ clade in fungi. Not surprisingly, metazoan PAQR1 proteins and fungal PQRA proteins form sister groups in the phylogenetic trees in Figs. 3 and 4, with a strong association in the latter (bootstrap value of 410/500 replicates) while metazoan PAQR3 proteins and fungal PQRB proteins also loosely associate in these trees. The simplest interpretation of these trees is that the PQRA and PQRB clades evolved before the split between fungi and metazoans and that the relationship between PAQR1/ PQRA and PAQR3/PQRB is one of homology. However, it may be equally likely that the HxxFH-type and HxxWH-type receptors represent distinct solutions to similar biochemical problems and that Fungi and Metazoa arrived at the same functional solutions through homoplasy.

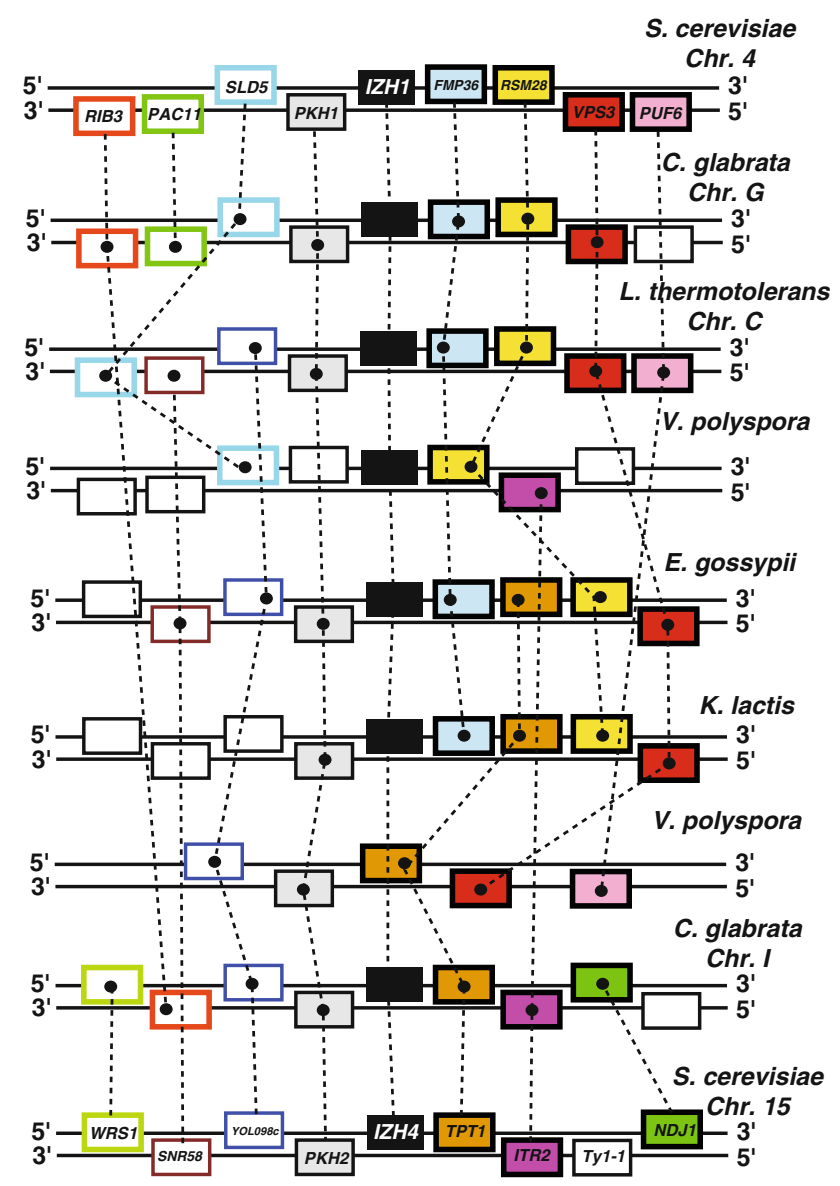

Fig. 7 Synteny of the genes surrounding IZHI and IZH4 on S. cerevisiae chromosomes 4 and 15 . This synteny is conserved in other yeasts that diverged after the whole genome duplication (WGD) event, including in C. glabrata and Vanderwaltozyma polyspora as well as pre-WGD yeast like Eremothecium gossypii, Kluyveromyces lactis, and Lachancea thermotolerans. Dashed lines connect homologous genes. S. cerevisiae gene names are indicated

Phylogenetic Analysis of the Fungal Class III PAQR Family

Nearly, all fungal genomes encode at least two Class I PAQRs and no Class III receptors. The only exceptions to this rule are species in the phylum Microsporidia, which possess Class III receptors but not Class I receptors. While, there is some debate about the placement of microsporidians among eukaryotic taxa, the general consensus is that microsporidians occupy the base of the fungal branch (James et al. 2006) and may actually form a sister group to fungi (Liu et al. 2006). Despite the fact that Class III receptors are highly divergent from other PAQRs, have no known function and are absent from most fungi, the fact that they are present in microsporidians means that the evolution of Class III receptors must be, at least briefly, discussed in this study. 

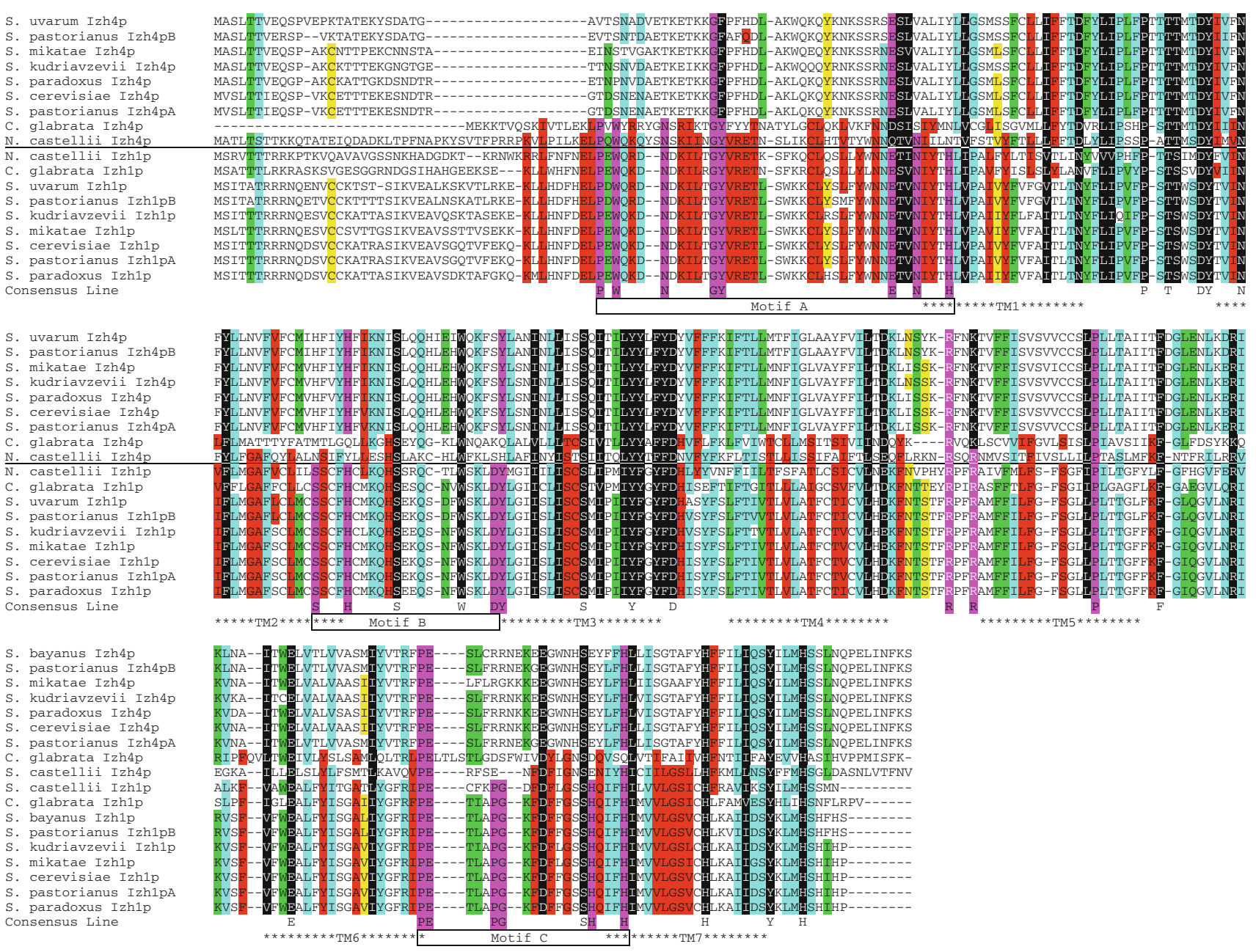

Fig. 8 Multiple sequence alignment of homologs of Izh1p and Izh4p from the "Saccharomyces complex". This multiple sequence alignment was produced manually. The shading key is as follows (\# of sequences out of 18 with conserved residue): red $\geq 10<12$, yellow $\geq 12<14$, green $\geq 14<16$, blue $\geq 16<18$, black 18 . Positions that are highly conserved in Classes I and II PAQRs, but not necessarily Class III PAQRs, are indicated with both purple shading and boxes

under the consensus line. Positions that are invariant in the above sequences are shown in the consensus line without shading. The following amino acid groupings are considered conserved: FY, EDQN, ILV, TS, PG, KR. Approximate location of predicted transmembrane domains are indicated below the consensus line (Color figure online)

Since, microsporidians are most likely highly derived fungi with contracted genomes due to their lifestyle as obligate intracellular parasites (Thomarat et al. 2004), it is reasonable to conclude that the progenitor of microsporidian lineage may have originally had Class I receptors, but they were eventually lost. It is less clear why Class III receptors are absent in non-microsporidian fungi since fungi are a sister group to holozoans, which do possess Class III receptors (excepting M. brevicollis) (see Fig. 3). This suggests that the ancestral opisthokont may have possessed Class III receptors, but they were lost in the fungal lineage at some point after the divergence of microsporidians.

However, phylogenetic trees of Class III receptors reveal that microsporidian PAQRs are more similar to apicomplexan Class III PAQRs (bootstrap value of 372/500

replicates) while those of holozoans cluster with those of other eukaryotes (bootstrap value of 410/500 replicates) (Fig. 3). Moreover, in trees containing eukaryotic and eubacterial Class III receptors, the eubacterial and metazoan Class III sequences cluster together (bootstrap value of 373/500 replicates), separate from the microsporidian, apicomplexan and, intriguingly, actinobacterial receptors (Fig. 11). Analysis of the probability-based consensuses for motifs A-C-in logo form-within each of the taxonomic groups in Fig. 11 (e.g., Actinobacteria, Apicomplexa, or Microsporidia) and for the entire Class III family strongly support the grouping of microsporidian receptors with those of apicomplexans and actinobacteria (Fig. 12). For the clade containing microsporidian, apicomplexan and actinobacterial receptors, the conserved asparagine in motif $\mathrm{A}$ is generally changed to arginine, while motif $\mathrm{C}$ can be 


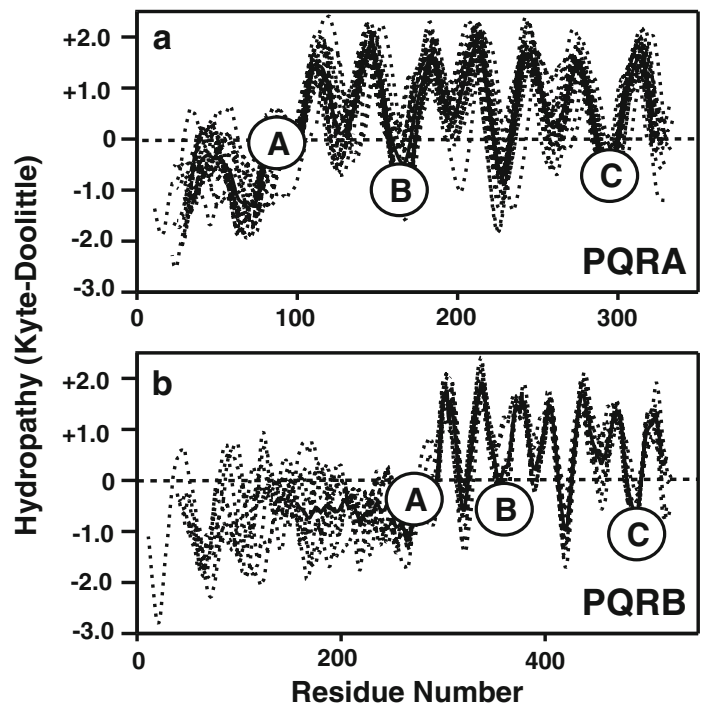

Fig. 9 Structural analysis of fungal PAQRs. Aligned and overlaid hydropathy plots for PAQRs from a variety of fungal species. Dotted lines indicate hydropathy plots for individual proteins. Bold lines indicate averaged hydropathy for all proteins in a particular group. Circled letters indicate the locations of motifs A-C that are conserved throughout the PAQR superfamily. a Proteins in the PQRA clade, b proteins in the PQRB clade

refined to a consensus of $\mathrm{HxxFH}$, with an invariant phenylalanine at position 4 of the motif. On the other hand, Class III receptors from metazoa generally conform to the whole-family consensus with the exception that motif $\mathrm{C}$ can be refined to a consensus of $\mathrm{HxxWH}$, with an invariant tryptophan at position 4 of the motif.

From this analysis, it is clear that microsporidian Class III receptors are not closely related to class III receptors from other opisthokont groups. This means that holozoan and microsporidian Class III receptors are probably not descended from the same ancestral opisthokont receptor. A simple explanation for this finding is that microsporidians do not belong in the opisthokont group and that they are more closely related to apicomplexans, however, we consider this possibility unlikely since most evidence suggests that microsporidians are-at the very least—close relatives of fungi. A more likely explanation for the presence of one type of Class III receptor in microsporidians, another type in holozoans and the absence of such receptors in other fungi is that Class III receptors were originally absent from the opisthokont ancestor and that microsporidian receptors were acquired by lateral gene transfer (LGT) from Actinobacteria or Apicomplexa while the holozoan receptors were acquired by a different LGT event. Alternatively, the opisthokont ancestor may have possessed both types of Class III receptor and that the "actinobacterial" type was lost in the branch leading to holozoans, while the "holozoan" type was lost in the branch leading to fungi and both receptors were lost in the fungal lineage after the divergence of Microsporidia. Indeed, the latter possibility may be more probable than the former double LGT, if one assumes that-all selective pressures being equal-it is easier to lose an useless gene by contraction than to acquire an useful one by LGT.

\section{HxxFH and HxxWH Clades}

Intriguingly, all Class III receptors either have a phenylalanine or a tryptophan at position 4 of motif $\mathrm{C}$ and Class III can be subcategorized into HxxFH or HxxWH subgroups. Thus, it appears as though the distinction between HxxFH-type and HxxWH-type receptors is present in Class III receptors as well as Class I receptors. Indeed, many species in the eubacterial phylum Firmicutes possess a receptor from each group, analogous to the distribution of receptor subtypes in fungi and metazoans. Thus, it is possible that the PQRA clade of Class I receptors arose from the HxxFH clade of Class III receptors, while the PQRB clade of Class I receptors arose from the HxxWH clade of Class III receptors. However, another equally reasonable interpretation of these findings is that the presence of distinct $\mathrm{HxxFH}$ and $\mathrm{HxxWH}$ clades in metazoans, fungi, and eubacteria is the result of homoplasy rather than homology. A third possibility is that the HxxFH and HxxWH groupings are merely coincidental and do not signify anything important. Much more study needs to be done to define the significance of these subtle differences in PAQR receptor sequence.

\section{Conclusions}

Using the powerful model system, S. cerevisiae, a better picture of the physiology of fungal PAQRs is beginning to emerge. Izh2p and Izh3p play an important role in the regulation of fungal development in response to external stimuli. In particular, their overexpression - which we have demonstrated to be functionally equivalent to their activation (Garitaonandia et al. 2009; Kupchak et al. 2007; Smith et al. 2008) - results in both inhibition of filamentous growth and increased sensitivity to polyene antifungal drugs.

Based on these findings and other published data, we conclude that fungal PAQRs represent exciting new potential antifungal targets. This conclusion is particularly salient for mycoses caused by $C$. glabrata, a post-WGD yeast that possesses a complement of PAQRs identical to that in S. cerevisiae. However, despite the widely held assumption that $S$. cerevisiae is harmless, $S$. cerevisiae strains are increasingly being isolated in clinical settings (Klingberg et al. 2008). Thus, the emergence of this species as an, albeit mild, opportunistic pathogen makes the results 

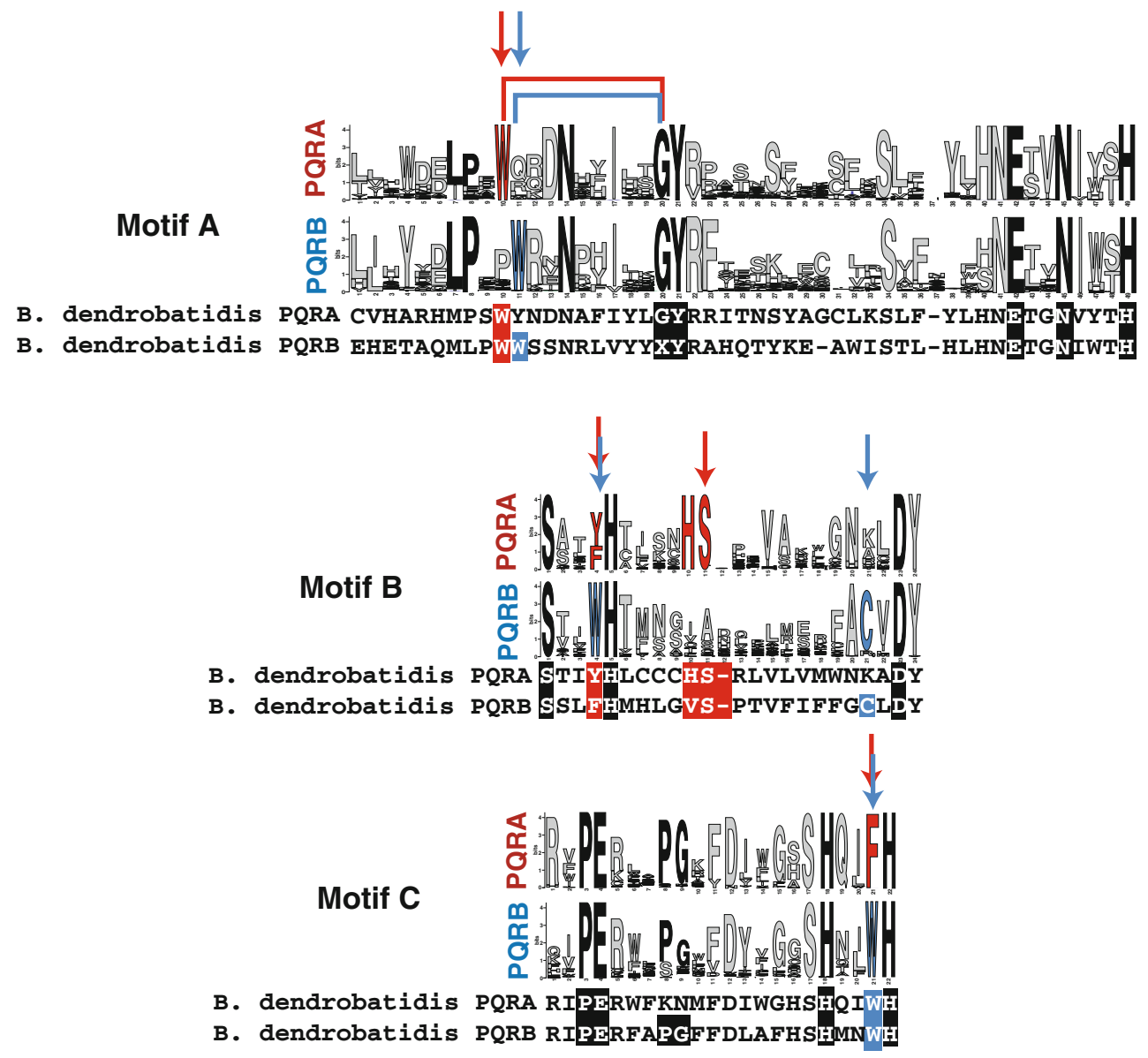

Fig. 10 Comparison of motifs $A-C$ in fungal PAQRs. Motifs A-C from the PQRA and PQRB clades presented in logo form produced by WebLogo. Consensus motifs were generated from all PQRA or PQRB homologs in the Supplementary data. Logos were imported into Adobe Illustrator to align the motifs and account for gaps in each motif. The height of the letters in each motif is proportional to the probability of finding that amino acid at that position of the motif. Arrows indicate positions that can be considered "diagnostic" of the

presented herein even more clinically relevant. Moreover, the fact that Izh2p and Izh3p are almost universally conserved in fungi suggests that they play a fundamental role in fungal physiology and that these results may be more broadly applicable to other, more distantly related fungi. The broad applicability of these findings is supported by the fact that the agonist for $S$. cerevisiae Izh2p, osmotin, is well known for its ability to prevent the growth of a wide array of taxonomically diverse pathogenic fungi. Indeed, the standard assay for measuring the toxicity of PR-5 proteins toward fungi actually does not measure cell viability, but rather the inhibition of hyphal growth on plates. Thus, it appears as though the relationship between PR-5 proteins, PAQRs and fungal development is also conserved across fungal taxa.

Since, developmental changes are integral for the virulence of many important fungal pathogens (Karkowska-Kuleta et al. two clades. Black letters indicate positions that are considered diagnostic of Class I PAQRs. Red and blue coloring indicates variations that are diagnostic of the PQRA and PQRB clades, respectively. PQRA and $\mathrm{PQRB}$ sequences from the chytrid fungus $B$. dendrobatidis are aligned under the consensus motifs. The letter $\mathrm{X}$ in the sequence of $\mathrm{PQRB}$ from $B$. dendrobatidis indicates that the amino acid at this position is unknown due to the fact that this is the location of a predicted intron-exon boundary (Color figure online)

2009), it is not surprising that there is already tremendous interest in PR-5 proteins as antifungal drugs. Unfortunately, PR-5 proteins are also potent allergens (Breiteneder 2004), making it unlikely that they could ever be used as pharmaceuticals. However, the identification of the PAQRs as plasma membrane receptors for PR-5 proteins opens up the possibility that small-molecule PR-5 mimics could be designed to target specific fungal Izh2p- or Izh3p-like proteins, essentially producing species-specific designer antifungal drugs. Such drugs would have the added benefit of acting extracellularly, thus greatly simplifying the pharmacodynamics. Novel agonists for these receptors could be used to inhibit fungal development or whichever fundamental physiological process they regulate. Moreover, our data suggest that PAQR agonists might also sensitize fungi to polyene antifungal agents. Such agonists could work synergistically with current antifungal regimens as adjuvants to 
Fig. 11 Phylogenetic analysis of fungal Class III PAQRs. A bootstrapped phylogenetic tree of select Class III PAQRs generated by the ML method. This tree shows the clustering of microsporidian proteins with those of apicomplexans and actinobacteria. Bootstrap values at the tree nodes are confidence values and indicate the number of times per 500 trees generated a particular grouping was made. Branch lengths are proportional to evolutionary distance and the scale bar indicates the number substitutions per site. Human and yeast Class I receptors are included as an outgroup

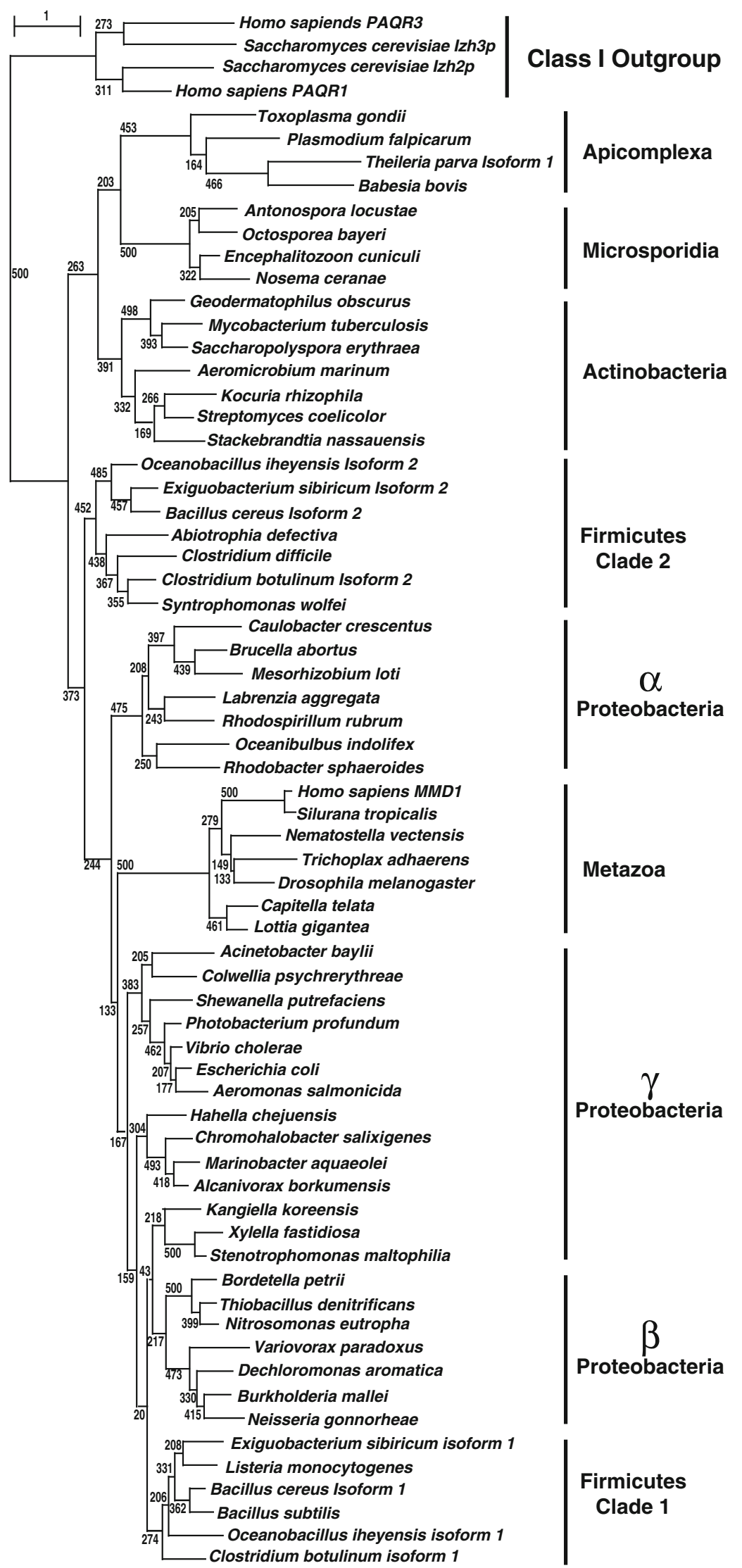


Fig. 12 Conserved motifs in Class III PAQRs. Motifs A-C from all members of each taxonomic grouping of Class III receptors and from all members of Class III included in the Supplementary data presented in logo form produced by WebLogo. Logos were imported into Adobe Illustrator to align the motifs and account for gaps in motif $\mathrm{B}$. The height of the letters in each motif is proportional to the probability of finding that amino acid at that position of the motif. $n$ refers to the number of sequences in each taxonomic grouping used to produce the individual motifs. Green letters indicate common deviations from the generalized motif shown at the bottom that can be considered characteristic of particular groups (e.g., the R at position 5 in motif $\mathrm{A}$ in Microsporidia, Apicomplexa, and Actinobacteria). Red and blue letters denote invariant tryptophan or phenylalanine residues in motif $\mathrm{C}$ that crudely separate Class III receptors into two distinct subgroups (Color figure online)

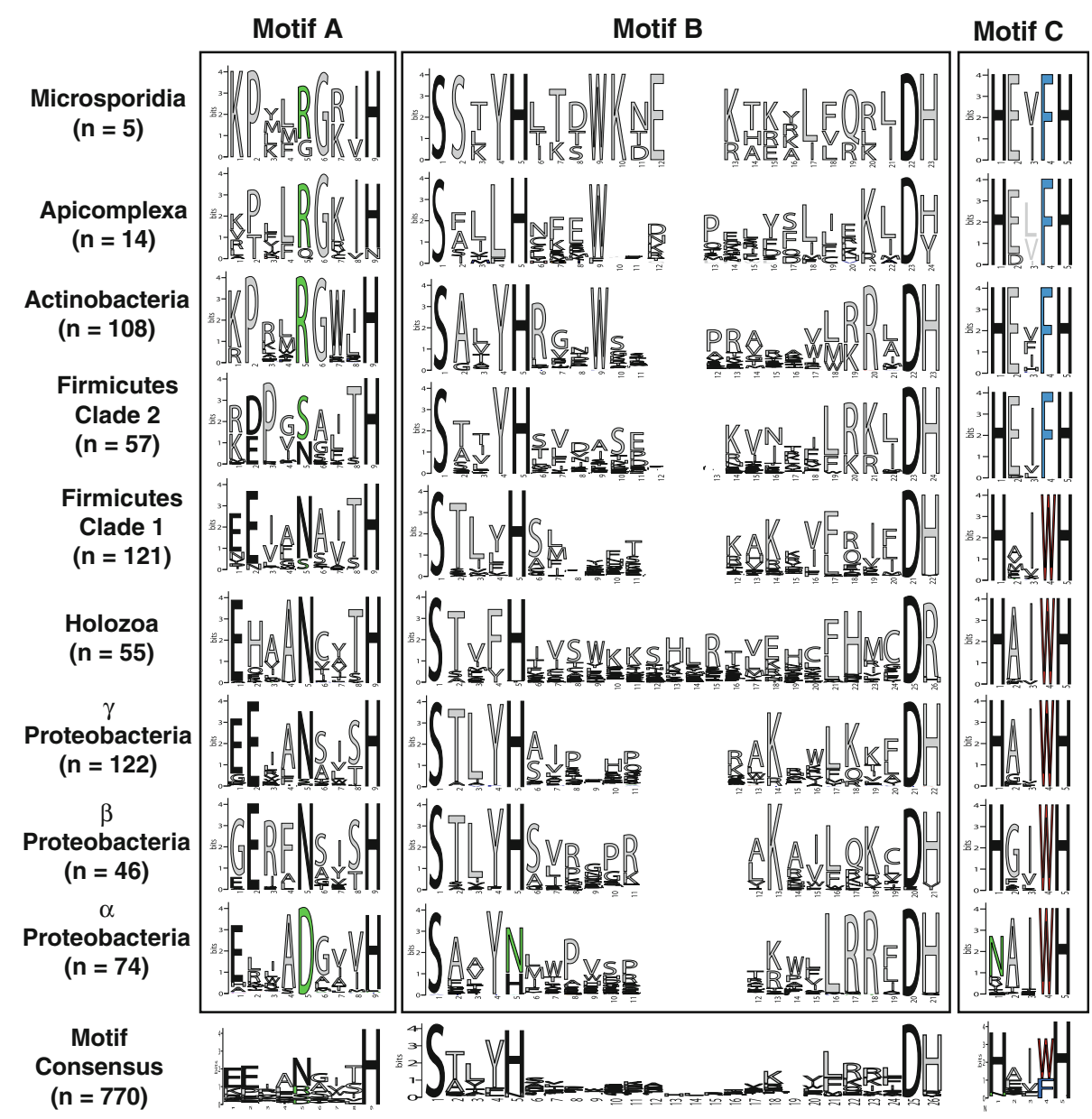

increase polyene potency or reduce dosages required to control recalcitrant infections.

Acknowledgments We would like to thank the NIH for funding R01 GM086617-01 and R21 DK074812 to TJL.

Open Access This article is distributed under the terms of the Creative Commons Attribution Noncommercial License which permits any noncommercial use, distribution, and reproduction in any medium, provided the original author(s) and source are credited.

\section{References}

Anzlovar S, Dermastia M (2003) The comparative analysis of osmotins and osmotin-like PR-5 proteins. Plant Biol 5:116-124

Baida GE, Kuzmin NP (1996) Mechanism of action of hemolysin III from Bacillus cereus. Biochim Biophys Acta 1284:122-124

Brauer AU, Nitsch R, Savaskan NE (2004) Identification of macrophage/microglia activation factor (MAF) associated with late endosomes/lysosomes in microglial cells. FEBS Lett 563:41-48

Breiteneder H (2004) Thaumatin-like proteins-a new family of pollen and fruit allergens. Allergy 59:479-481

Chabasse D (1998) Origin and interrelation of Fungi with the living. Evolution in geologic times. J Mycol Med 8:125-138

Chen YC, Chang MC, Chuang YC, Jeang CL (2004) Characterization and virulence of hemolysin III from Vibrio vulnificus. Curr Microbiol 49:175-179
Claros MG, von Heijne G (1994) TopPred II: an improved software for membrane protein structure predictions. Comput Appl Biosci 10:685-686

Crooks GE, Hon G, Chandonia JM, Brenner SE (2004) WebLogo: a sequence logo generator. Genome Res 14:1188-1190

Edgar RC (2004) MUSCLE: multiple sequence alignment with high accuracy and high throughput. Nucl Acids Res 32:1792-1797

Ferreira RB, Monteiro S, Freitas R, Santos CN, Chen Z, Batista L et al (2007) The role of plant defence proteins in fungal pathogenesis. Mol Plant Path 8:677-700

Fitzpatrick DA, Logue ME, Stajich JE, Butler G (2006) A fungal phylogeny based on 42 complete genomes derived from supertree and combined gene analysis. BMC Evol Biol 6:15

Garitaonandia I, Smith JL, Kupchak BR, Lyons TJ (2009) Adiponectin identified as an agonist for PAQR3/RKTG using a yeastbased assay system. J Recept Signal Transduct 29:67-73

Goldstein AL, McCusker JH (1999) Three new dominant drug resistance cassettes for gene disruption in Saccharomyces cerevisiae. Yeast 15:1541-1553

Gordon JL, Byrne KP, Wolfe KH (2009) Additions, losses, and rearrangements on the evolutionary route from a reconstructed ancestor to the modern Saccharomyces cerevisiae genome. PLoS Genet 5:e1000485

Gouy M, Guindon S, Gascuel O (2010) SeaView version 4: a multiplatform graphical user interface for sequence alignment and phylogenetic tree building. Mol Biol Evol 27:221-224

Guindon S, Delsuc F, Dufayard JF, Gascuel O (2009) Estimating maximum likelihood phylogenies with PhyML. Methods Mol Biol 537:113-137 
James TY, Kauff F, Schoch CL, Matheny PB, Hofstetter V, Cox CJ et al (2006) Reconstructing the early evolution of Fungi using a six-gene phylogeny. Nature 443:818-822

Jin R, Dobry CJ, McCown PJ, Kumar A (2008) Large-scale analysis of yeast filamentous growth by systematic gene disruption and overexpression. Mol Biol Cell 19:284-296

Karkowska-Kuleta J, Rapala-Kozik M, Kozik A (2009) Fungi pathogenic to humans: molecular bases of virulence of Candida albicans, Cryptococcus neoformans and Aspergillus fumigatus. Acta Biochim Pol 56:211-224

Karpichev IV, Cornivelli L, Small GM (2002) Multiple regulatory roles of a novel Saccharomyces cerevisiae protein, encoded by YOLO02c , in lipid and phosphate metabolism. J Biol Chem 277:19609-19617

Kellis M, Patterson N, Endrizzi M, Birren B, Lander ES (2003) Sequencing and comparison of yeast species to identify genes and regulatory elements. Nature 423:241-254

Klingberg TD, Lesnik U, Arneborg N, Raspor P, Jespersen L (2008) Comparison of Saccharomyces cerevisiae strains of clinical and nonclinical origin by molecular typing and determination of putative virulence traits. FEMS Yeast Res 8:631-640

Kulkarni RD, Thon MR, Pan H, Dean RA (2005) Novel G-proteincoupled receptor-like proteins in the plant pathogenic fungus Magnaporthe grisea. Genome Biol 6:R24

Kupchak BR, Garitaonandia I, Villa NY, Mullen MB, Weaver MG, Regalla LM et al (2007) Probing the mechanism of FET3 repression by Izh2p overexpression. Biochim Biophys Acta Mol Cell Res 1773:1124-1132

Kupchak BR, Villa NY, Kulemina LV, Lyons TJ (2008) Dissecting the regulation of yeast genes by the osmotin receptor. Biochem Biophys Res Commun 374:210-213

Kurtzman CP (2003) Phylogenetic circumscription of Saccharomyces, Kluyveromyces and other members of the Saccharomycetaceae, and the proposal of the new genera Lachancea, Nakaseomyces, Naumovia, Vanderwaltozyma and Zygotorulaspora. FEMS Yeast Res 4:233-245

Lafon A, Han KH, Seo JA, Yu JH, d'Enfert C (2006) G-protein and cAMP-mediated signaling in aspergilli: a genomic perspective. Fungal Genet Biol 43:490-502

Libkind D, Hittingerb CT, Valériod E, Gonçalves C, Doverb J, Johnston $M$ et al (2011) Microbe domestication and the identification of the wild genetic stock of lager-brewing yeast. Proc Natl Acad Sci USA 108:14539-14544

Liu YJ, Hodson MC, Hall BD (2006) Loss of the flagellum happened only once in the fungal lineage: phylogenetic structure of kingdom Fungi inferred from RNA polymerase II subunit genes. BMC Evol Biol 6:74

Lopes SC, Goormaghtigh E, Cabral BJ, Castanho MA (2004) Filipin orientation revealed by linear dichroism. Implication for a model of action. J Am Chem Soc 126:5396-5402

Lynch M, Conery JS (2000) The evolutionary fate and consequences of duplicate genes. Science 290:1151-1155

Lyons TJ, Villa NY, Regalla LM, Kupchak BR, Vagstad A, Eide DJ (2004) Metalloregulation of yeast membrane steroid receptor homologs. Proc Natl Acad Sci USA 101:5506-5511

Massey SE, Moura G, Beltrao P, Almeida R, Garey JR, Tuite MF et al (2003) Comparative evolutionary genomics unveils the molecular mechanism of reassignment of the CTG codon in Candida spp. Genome Res 13:544-557

Miura F, Kawaguchi N, Sese J, Toyoda A, Hattori M, Morishita S et al (2006) A large-scale full-length cDNA analysis to explore the budding yeast transcriptome. Proc Natl Acad Sci USA 103:17846-17851

Murad AM, Leng P, Straffon M, Wishart J, Macaskill S, MacCallum D et al (2001) NRG1 represses yeast-hypha morphogenesis and hypha-specific gene expression in Candida albicans. EMBO J 20:4742-4752

Narasimhan ML, Coca MA, Jin J, Yamauchi T, Ito Y, Kadowaki T et al (2005) Osmotin is a homolog of mammalian adiponectin and controls apoptosis in yeast through a homolog of mammalian adiponectin receptor. Mol Cell 17:171-180

Rehli M, Krause SW, Schwarzfischer L, Kreutz M, Andreesen R (1995) Molecular cloning of a novel macrophage maturationassociated transcript encoding a protein with several potential transmembrane domains. Biochem Biophys Res Commun 217:661-667

Robertson KP, Smith CJ, Gough AM, Rocha ER (2006) Characterization of Bacteroides fragilis hemolysins and regulation and synergistic interactions of HlyA and HlyB. Infect Immun 74:2304-2316

Scannell DR, Frank AC, Conant GC, Byrne KP, Woolfit M, Wolfe $\mathrm{KH}$ (2007) Independent sorting-out of thousands of duplicated gene pairs in two yeast species descended from a whole-genome duplication. Proc Natl Acad Sci USA 104:8397-8402

Smith JL, Kupchak BR, Garitaonandia I, Hoang LK, Maina AS, Regalla LM et al (2008) Heterologous expression of human $\operatorname{mPR} \alpha, \operatorname{mPR} \beta$ and $\operatorname{mPR} \gamma$ in yeast confirms their ability to function as membrane progesterone receptors. Steroids 73:11601173

Tang YT, Hu T, Arterburn M, Boyle B, Bright JM, Emtage PC et al (2005) PAQR proteins: a novel membrane receptor family defined by an ancient 7-transmembrane pass motif. J Mol Evol 61:372-380

Taylor JW, Berbee ML (2006) Dating divergences in the fungal tree of life: review and new analyses. Mycologia 98:838-849

te Welscher YM, ten Napel HH, Balague MM, Souza CM, Riezman $\mathrm{H}$, de Kruijff B et al (2008) Natamycin blocks fungal growth by binding specifically to ergosterol without permeabilizing the membrane. J Biol Chem 283:6393-6401

Thomarat F, Vivares CP, Gouy M (2004) Phylogenetic analysis of the complete genome sequence of Encephalitozoon cuniculi supports the fungal origin of microsporidia and reveals a high frequency of fast-evolving genes. J Mol Evol 59:780-791

Villa NY, Kupchak BR, Garitaonandia I, Smith JL, Alonso E, Alford $\mathrm{C}$ et al (2009) Sphingolipids function as downstream effectors of a fungal PAQR. Mol Pharmacol 75:866-875

Wach A, Brachat A, Pohlmann R, Philippsen P (1994) New heterologous modules for classical or PCR-based gene disruptions in Saccharomyces cerevisiae. Yeast 10:1793-1808

Whelan S, Goldman N (2001) A general empirical model of protein evolution derived from multiple protein families using a maximum-likelihood approach. Mol Biol Evol 18:691-699

Yamauchi T, Kamon J, Ito Y, Tsuchida A, Yokomizo T, Kita S et al (2003) Cloning of adiponectin receptors that mediate antidiabetic metabolic effects. Nature 423:762-769

Yoshida S, Hashimoto K, Shimada E, Ishiguro T, Minato T, Mizutani $S$ et al (2007) Identification of bottom-fermenting yeast genes expressed during lager beer fermentation. Yeast 24:599-606

Zhu Y, Bond J, Thomas P (2003) Identification, classification, and partial characterization of genes in humans and other vertebrates homologous to a fish membrane progestin receptor. Proc Natl Acad Sci USA 100:2237-2242 